\title{
GCU
}

Glasgow Caledonian

University

University for the Common Good

\section{On the design and deployment of multitier heterogeneous and adaptive vehicular networks}

Ansari, Shuja; Boutaleb, Tuleen; Sinanovic, Sinan; Gamio, Carlos; Krikidis, loannis

Published in:

2018 11th International Symposium on Communication Systems, Networks and Digital Signal Processing, CSNDSP 2018

DOI:

10.1109/CSNDSP.2018.8471807

Publication date:

2018

Document Version

Author accepted manuscript

Link to publication in ResearchOnline

Citation for published version (Harvard):

Ansari, S, Boutaleb, T, Sinanovic, S, Gamio, C \& Krikidis, I 2018, On the design and deployment of multitier heterogeneous and adaptive vehicular networks. in 201811 th International Symposium on Communication Systems, Networks and Digital Signal Processing, CSNDSP 2018., 8471807, IEEE.

https://doi.org/10.1109/CSNDSP.2018.8471807

\section{General rights}

Copyright and moral rights for the publications made accessible in the public portal are retained by the authors and/or other copyright owners and it is a condition of accessing publications that users recognise and abide by the legal requirements associated with these rights.

Take down policy

If you believe that this document breaches copyright please view our takedown policy at https://edshare.gcu.ac.uk/id/eprint/5179 for details

of how to contact us. 


\title{
On the Design and Deployment of Multitier Heterogeneous and Adaptive Vehicular Networks
}

\author{
Shuja Ansari ${ }^{1}$, Tuleen Boutaleb ${ }^{1}$, Sinan Sinanovic ${ }^{1}$, Carlos Gamio ${ }^{1}$ and Ioannis Krikidis ${ }^{2}$ \\ ${ }^{1}$ School of Engineering and Built Environment, Glasgow Caledonian University, Glasgow, Scotland UK \\ Email: Shuja.Ansari, T.Boutaleb, Sinan.Sinanovic, Carlos.Gamio@gcu.ac.uk \\ ${ }^{2}$ Department of Electrical and Computer Engineering, Faculty of Engineering, University of Cyprus \\ Email: krikidis@ucy.ac.cy
}

\begin{abstract}
Research on connected and autonomous vehicles (CAVs) is moving towards first deployments around the world. For complete vehicle autonomy, on top of sensors there is a need for an effective communication system. Due to the critical safety, transmission requirements for these communications are stringent. In an urban environment, with high density of vehicles, standardized dedicated short range communications (DSRC) solely does not perform well. Avoiding costs for new DSRC infrastructure, heterogeneous networks integrating long term evolution (4G-LTE mobile network) and DSRC have shown promising results. With the ever increasing number of vehicles, an optimal integration is required in order to balance the capacity load on the two networks. This paper proposes a systematic approach to designing multitier heterogeneous adaptive vehicular (MHAV) networks. With extensive system level simulations modeling Glasgow city center, incorporating proposed algorithms, scaling of the network along with load balancing between LTE and DSRC have been investigated in this paper. With the design criteria proposed for MHAV, results show that under realistic conditions the probability of end-to-end communication delay to be less than $50 \mathrm{~ms}$ is above $90 \%$ for a density of 250 vehicles $/ \mathbf{k m}^{2}$.
\end{abstract}

\section{INTRODUCTION}

Connected and autonomous vehicles (CAVs) have seen tremendous research efforts in the last decade. The Society of automotive engineers (SAE) have drafted a standard pertaining five levels of vehicle automation [1]. Level-0 (L-0) states no automation where the driver performs full-time dynamic driving tasks. L-1 drive assistance automates either steering or acceleration/deceleration using information about the driving environment. L-2 brings in simultaneous automation in both steering and acceleration/deceleration to provide cruisecontrol and lane change assistance while the human driver performs all the remaining aspects of dynamic driving. Pure automation starts from L-3, where the driving is performed by automated driving system under controlled environment like straight highways. L-4 advances the automated driving systems to perform all the aspects of dynamic driving under all pre-mapped scenarios. Between L-3 and L-4, the driver is required to respond appropriately (in time) to a request to intervene. Finally, L-5 states full automation, where the presence of steering, accelerator, brakes, etc. are not required in the vehicle.

Automation up until L-3 and some driving modes of L-4 is achievable using sensors or cameras on board the vehicle. However, according to [2]-[4], L-4 and above automation requires an efficient vehicular communication network. These communications pertaining CAVs are also a big part of cooperative intelligent transportation systems (C-ITS) where, human safety is vital among other applications. Exchange of information is carried out in the form of vehicle-to-vehicle
$(\mathrm{V} 2 \mathrm{~V})$, vehicle-to-infrastructure (V2I), vehicle-to-pedestrian (V2P), all collectively termed vehicle-to-everything (V2X) communications. Essential communications for vehicle safety at L-4 and above automation requires the use of heterogeneous networks [4]. These are multi-radio networks integrating dedicated short range communications (DSRC) and mobile networks such as long term evolution (LTE), evolving the $4 \mathrm{G}$ and $5 \mathrm{G}$ technologies. A number of LTE performance evaluations for the feasibility of use with vehicular ad hoc networks (VANETs) have suggested significant suitability, however, without any centralization; VANETs can pose enormous network capacity issues on the cellular network [5]. With the global mobile data traffic increasing sevenfold between 2016 and 2021 [6], the availability of spectrum for VANETs can be uncertain.

In terms of using only DSRC for vehicular communications, low latency is experienced as compared to LTE, however, a new infrastructure is required for V2I communications. Successful message delivery in highly dense urban scenarios is also not evident [7]. For the centralization of DSRC, there are some proposed techniques and frameworks. Among these, clustering and various routing protocols [8] are some of the promising DSRC techniques. However, with clustering or direct vehicular communication, the concern of privacy and security arises.

For the purpose of VANET centralization on LTE, group formation, multicast/broadcast management system (MBMS) and device-to-device (D2D) communications have been proposed [9], [10]. Group formation also known as clustering has shown promising performance. However, according to [11], $35 \%$ of road users are concerned about privacy in regards to sharing their information with other road users. At the same time, clustering relies on relaying transmissions which can pose a privacy and security issue [12]. MBMS functionality also proved to be reliable for message dissemination, although being part of 3GPP specifications, MBMS is not widely implemented by mobile network operators (MNOs) [13]. Similarly, D2D communications also referred to as LTE direct communications, using full duplex radios in order to enable vehicles to receive and transmit at the same time, have shown reduction in the use of LTE uplink resources, increasing network capacity. However, D2D for VANETs exhibits an increase in interference [10] and similar to MBMS, is not currently implemented by MNOs.

Due to the frequent and fixed routes of public transit, studies have suggested the use of buses as mobile gateways (MG) instead of fixed road-side units (RSUs) [14]-[17]. Many advantages such as their tall structure exhibiting higher 
transmission range, covering most parts of urban areas, no requirement of privacy mechanisms and avoiding the cost of installing a new infrastructure, make public buses a good substitute for fixed base stations. This paper builds upon our previous work that proposes a multitier framework [18], where authority owned or public transport are high tier nodes (HTN) acting as MGs, incorporating message dissemination scheme proposed in [19] with a fallback mechanism and MG registration technique proposed in [20].

With the technical aspects of the proposed architecture including efficient message forwarding, robust registration scheme and reliable fall back mechanism in place, this paper includes a systematic approach to the design and deployment of multitier heterogeneous adaptive VANETs (MHAV) in urban environments is proposed. Contributions of this paper include:

- Design criteria for a multitier heterogeneous and adaptive vehicular network (MHAV).

- Performance evaluation of MHAV under realistic urban environment with high density of vehicles employing mutlicell and multipath channel fading models.

The remainder of this paper is organized as follows: Section II describes the design criteria for multitier heterogeneous framework, and Section III elaborates on the system model followed by simulation results in Section IV. Conclusions and future work are then discussed within Section V.

\section{Multitier Heterogeneous Adaptive VANETs}

The proposed multitier heterogeneous adaptive VANET (MHAV) framework incorporates high tier nodes (HTN) and low tier nodes (LTN). HTNs are the authority owned vehicles such as public buses, taxis, council lorries, etc. while LTNs comprise all the other private vehicles. Both HTNs and LTNs are assumed to be equipped with LTE and DSRC interface, integrated with the help of a control device.

Data delivery in the proposed framework is carried out with the cooperation of HTNs, traffic control center (TCC) and vehicular safety application (VSA) server. The TCC and VSA are situated at the core of mobile network. All the LTNs get registered with HTNs, which then enables V2I communications. If an HTN is not available, LTN falls back to using LTE network. HTNs consistently communicate with the LTE network, updating the traffic conditions and their registered LTNs table with the TCC and VSA. HTNs broadcast beacons every second consisting of their location, velocity and ID using DSRC. LTNs receiving these broadcasts run the registration algorithm in order to register with the most suitable HTN. Once the LTN is registered, all V2V communications are carried out via the registered HTN, acting as a message relay. The basic architecture of MHAV framework is shown in Fig. 1.

Since all the traffic related information is updated in the TCC, LTNs not registered with HTNs can also access this information via the mobile network. In regards to safety applications, we suggest the use of a differentiated quality of service (QoS) mechanism known as safety application identifier (SAI) proposed in [19] and implemented via the VSA server. In the next subsection, the multi-radio access technology (RAT) network is elaborated followed by the HTN selection algorithm which is implemented at the LTN. Furthermore, MHAV framework has a number of benefits over other previous similar approaches such as no clustering requirement, more efficient adaptation, higher applicability, increased security, better network load balancing, etc.

\section{A. Multitier Multi-RAT Network}

The proposed network is multitiered in terms of high and low tier nodes, however, the radio technologies employed are integrated and assumed to be present in all vehicles. DSRC operating at the $5.9 \mathrm{GHz}$ band with carrier sense multiple access with collision avoidance (CSMA/CA) faces difficulties when it comes to highly dense urban areas. DSRC frequency band is also found not to be suitable for NLOS conditions at intersections due to buildings and foliage [21]. On the other hand, LTE operating at lower frequency bands than DSRC, is found to be suitable for vehicular communications. However, due to scalability and capacity issues, mobile networks with an ever increasing number of users, might not be able to accommodate the vehicular networks. As mentioned earlier, vehicle safety have stringent requirements in terms of latency. Therefore, need for a multi-RAT system, overcoming shortfalls of independent RAT, is in place.

The most important requirement is the latency of message delivery. For a human driver, the stopping distance for when a hazard happens is the sum of driver thinking distance and actual braking distance [22]. The thinking distance is the time for a driver to react to a situation. For an autonomous vehicle, message must be delivered during the thinking distance time, in order to prevent an accident. For a vehicle driving at 30 miles per hour, stopping distance is $23 \mathrm{~m}$ where $9 \mathrm{~m}$ is the thinking distance and the remaining $14 \mathrm{~m}$ is the actual braking distance. Keeping this in mind for urban environments, using the time, distance and speed relationship, the time an autonomous car will have to make a decision would be less than $670 \mathrm{~ms}$. However, if the speed increases, the breaking distance increases while decreasing the thinking time.

Apart from these driving rules, the standards require a latency of less than $100 \mathrm{~ms}$ [23] while literature has benchmarked it at $50 \mathrm{~ms}$ for successful implementation of vehicular networks [24], [25]. With these driving rules and the standard requirements, a suitable RAT is to be determined. For our proposed framework, we find DSRC to be effective in the low tier, however, with no presence of HTN, latency requirement can also be met by LTNs communicating over LTE, provided the mobile network is not saturated.

\section{B. HTN Selection Algorithm}

By having HTNs with integrated DSRC and LTE, a system is designed where the HTNs act as gateways and message relays. In order to choose an HTN, LTNs run our proposed algorithm every time a broadcast beacon is received. Similar to the scheme proposed in [14], each HTN maintains a registration table recording the LTNs currently registered with them. These tables are constantly reported and updated by TCC over the LTE network. In order to have a robust network, especially with such a mobile topology, determining which HTN to select for registration is an important issue when LTNs can receive multiple broadcast beacons from a number of HTNs.

When an LTN receives a broadcast beacon from HTN, this node is placed in the candidate registration set $(S)$. Using the information in the broadcasted beacon, LTN calculates the connection delivery delay $(T)$ for every HTN in the 


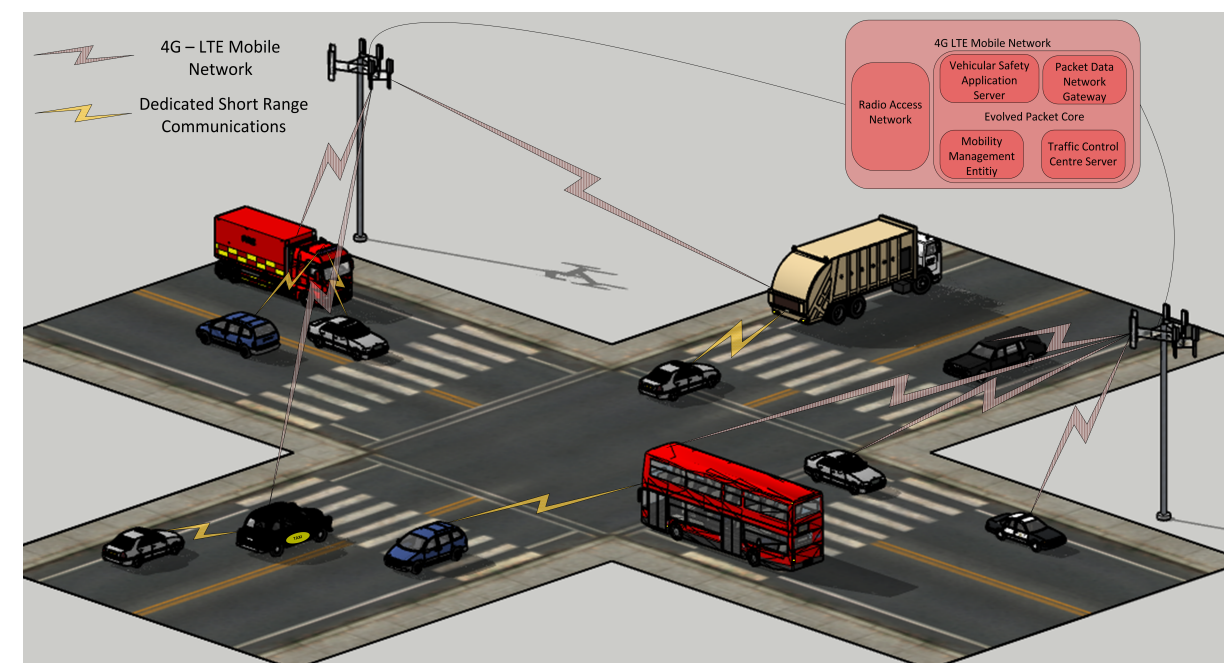

Fig. 1. Multitier Heterogeneous Adaptive VANET Framework showing HTNs and LTNs with possible scenarios.

candidate registration set. This delay is calculated using HTN's predefined transmission range $(R)$, distance between the $\mathrm{HTN}_{j}$ and $\operatorname{LTN}_{i}\left(d_{i j}\right)$ and their relative velocity $\left(v_{i}-v_{j}\right)$. Negative value of this delay means that the HTN is moving in the opposite direction to the LTN, therefore if $T$ is negative the HTN is placed in the discard set. Out of all the HTNs residing in $S$, the one with the highest $T$ is selected for registration. Once LTN has registered with the HTN, it stays connected with it while the distance between LTN and HTN remains below $R$. Proposed registration algorithm is elaborated in [20].

Setting a threshold and employing multi-hop approach avoids ping-pong effect but results in high delays [14]. To tackle this ping-pong problem in our evaluations, we preset $R$ and force the LTN to stay connected with the registered HTN until it moves out of the transmission range. To select an optimum $R$, the system is tested under varying values of $R$. The results showed a trade-off between number of registration switches and DSRC coverage area for urban environment. Once the LTN is aware of its forwarding via the chosen HTN, message dissemination can be carried out accordingly. Different aspects and scenarios for message dissemination are discussed in the following subsection.

\section{Message Dissemination Scenarios and Network Scalability}

Successful message delivery is vital for vehicular safety applications, hence, the designed framework must maintain connectivity at all times. Network scalability and redundancy play a vital role along with robust adaptive message dissemination schemes. In terms of network scalability and message dissemination, VSA server placement near the edge of the network is necessary. Considering the data freshness concept, ideal location of the server would be at the EPC. Therefore, having multiple EPCs serving their respective geographical locations would have their own VSA servers with a similar approach as is for mobility management entity (MME), reducing the RTT while increasing the system capacity and eventually meeting the strict transmission requirements for vehicular safety applications [26].

Recent 3GPP release 15, outlining 5G mobile networks, proposes network slicing in order to make the network flexible and scalable [27]. 5G is considered to be an evolution of LTE, where rel-15 currently specifies the migration procedures to 5G. Network slicing deals with the scalability problem specially for vehicular communications over mobile networks. Initial concept for network slicing proposes virtualization of networks serving dedicated applications such as vehicular networks, mobile broadband, voice and video applications, and so on. For the remaining of the paper, results and discussions are included with respect to the following scenarios in an urban environment.

1) Scenario-I: HTN - DSRC Message Dissemination: As mentioned earlier, V2V communications take place via the selected HTN. This type of communication employs DSRC to enable low latency message forwarding. Once an $\mathrm{LTN}_{i}$ is registered with an $\mathrm{HTN}_{x}$, it will start sending periodic messages to the $\mathrm{HTN}_{x}$. This HTN will then look up the routing tables and determine the forwarding set as:

$$
\mathbf{F}_{i}=\left\{\forall k: d_{i k}<R_{i}, i \neq k\right\},
$$

where $R_{i}$ is the awareness range, $d_{i k}$ is the distance from $\mathrm{LTN}_{i}$ to the neighboring $\mathrm{LTN}_{k}$. Recalling the concept of safety application indexing [19], depending on the application, the $\mathrm{HTN}_{x}$ will adapt to the respective $R_{i}$ and beacon frequency for the particular message type sent by the $\mathrm{LTN}_{i}$. In the case, where a receiving $\mathrm{LTN}_{k}$ is registered with another $\mathrm{HTN}_{y}$, the tables populated and updated by TCC are looked up to determine whether the $\mathrm{HTN}_{y}$ is in the transmission range of $\mathrm{HTN}_{x}$. If the latter is not in the transmission range, then the mobile network along with TCC server forwards the message to the respective HTN.

2) Scenario-II: HTNs Offloading LTE: Having HTNs serve LTN communications, addresses the privacy issue while providing low latency communications and balancing the load on the two radio networks. In this scenario, we simulate the entire network while evaluating the performance and load on LTE network. For designing purposes, we determine the ratio of HTNs to LTNs in order to provide efficient safety communications. With this ratio, load balancing between DSRC and LTE is evaluated.

3) Scenario-III: LTE Message Dissemination: If an HTN does not fulfill the criteria described in [20], it will result in the LTN being disconnected from the DSRC network. To ensure full time connectivity we propose a fall-back to using the LTE mobile network. Previous evaluations show that the LTE 


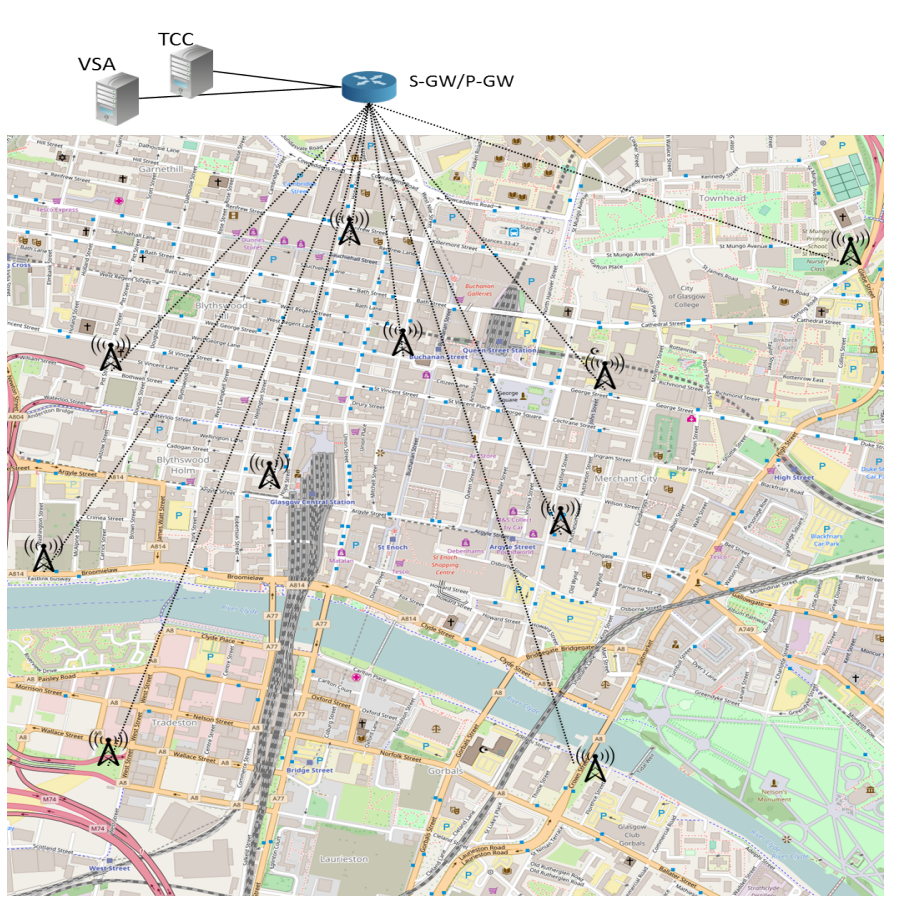

Fig. 2. $2 \times 2 \mathrm{~km}^{2}$ area of Glasgow city center covered by 10 sites with 3 cells/site.

network can accommodate a low density of vehicles provided a resource allocation scheme is in place [26]. Therefore, in a high density network, if a certain ratio of LTNs are offloaded to DSRC, the LTE mobile network can substantially meet the transmission requirements. This is further explained in Section IV.

\section{SySTEM MODEL}

The network modeled is a $2 \times 2 \mathrm{~km}^{2}$ area of Glasgow city center with varying density of vehicles evaluating both rush hours when there is high presence of HTNs and less busy hours with less HTNs available. Both LTNs and HTNs are assumed to be equipped with FDD LTE transceivers with $20 \mathrm{MHz}$ bandwidth, uplink carrier frequency $1715 \mathrm{MHz}$ and downlink carrier frequency $2115 \mathrm{MHz}$ (band 4) [28, Table 5.51] integrated with IEEE $802.11 \mathrm{p}$ compliant DSRC interface operating at $5.9 \mathrm{GHz}$ with $10 \mathrm{MHz}$ bandwidth. These nodes are assumed to be moving in urban model created using routes mobility model [29]. Fig. 2 illustrates the service area modeled in ns-3 [30]. Nodes move at an urban average speed matched to the 3GPP extended vehicular A (EVA) model radio environment designed using MATLAB [31]. Simulation parameters used are given in Table I.

Furthermore for HTNs, predefined bus routes are modeled with an interval of 10 minutes. For the eNodeBs (eNBs), mast data for operator EE has been implemented. The eNBs are connected to the mobility management entity (MME) through their S1-AP interface and to the serving gateway (S-GW) and packet data network gateway (P-GW) through their S1-U interfaces. Interconnection from the P-GW to the TCC Server and VSA server is modeled using an error free $10 \mathrm{Gbps}$ pointto-point link and TCP/IP version 4. The packet payload for HTNs is assumed to be 1500 bytes including the registration tables, locations and safety application data.

Propagation loss model employed for IEEE 802.11p is Nakagami loss model with the path loss factor $(m)$ of 4 on
TABLE I

SimULATION PARAMETERS

\begin{tabular}{|c|c|}
\hline Parameter & Value \\
\hline Simulation time & 300 seconds. \\
\hline Road model & $2 \times 2 \mathrm{~km}^{2}$ Glasgow City Center \\
\hline Number of LTNs & 1000,2000 vehicles $/ \mathrm{km}^{2}$. \\
\hline Ratio of HTNs to LTNs & $0, \frac{1}{10}, \frac{3}{10}, \frac{5}{10}$. \\
\hline Average speed & $20 \mathrm{mph}(\mathrm{HTN}), 30 \mathrm{mph}$ (LTN). \\
\hline \multicolumn{2}{|l|}{ DSRC } \\
\hline Access Technology & IEEE $802.11 \mathrm{p}$. \\
\hline Propagation model & Nakagami-m and Log distance Models. \\
\hline Operating Frequency & $5.9 \mathrm{GHz}$. \\
\hline Data Rate & $6 \mathrm{Mbps}$. \\
\hline Transmission Power & $25 \mathrm{dBm}$. \\
\hline Antenna & Omnidirectional. \\
\hline Channel Bandwidth & $10 \mathrm{MHz}$. \\
\hline Noise Figure & $7 \mathrm{~dB}$. \\
\hline CCA threshold & $-86 \mathrm{dBm}$ \\
\hline \multicolumn{2}{|l|}{ LTE } \\
\hline Network & 10 sites with 3 cells/site. \\
\hline Transmission power & eNB: $40 \mathrm{dBm}$, UE: $23 \mathrm{dBm}$. \\
\hline Carrier frequency DL/UL & $2115 \mathrm{MHz} / 1715 \mathrm{MHz}$ \\
\hline Channel bandwidth & $20 \mathrm{MHz}(100 \mathrm{RBs})$ \\
\hline Noise Figure & eNB: $5 \mathrm{~dB}$, UE: $9 \mathrm{~dB}$. \\
\hline UE antenna model & Isotropic $(0 \mathrm{dBi})$. \\
\hline eNB antenna model & $15 \mathrm{~dB}$ Cosine model, $65^{\circ} \mathrm{HPBW}$. \\
\hline Scheduling algorithm & Proportional Fair. \\
\hline Handover algorithm & $\begin{array}{l}\text { A2A4RSRQ, RSRQ threshold }-5 \mathrm{~dB} \text {, } \\
\text { and NeighbourCellOffset }=2(1 \mathrm{~dB}) \text {. }\end{array}$ \\
\hline Path loss model & LogDistance $(\alpha=3)$ and \\
\hline & 3GPP Extended Vehicular A model. \\
\hline
\end{tabular}

top of Friis propagation loss model. Results are obtained by scaling the modeled network. An area of $200 \times 200 \mathrm{~m}^{2}$ is first modeled with 10 vehicles, then the network is scaled with a linear scale factor (SF) of 2 and 3 to areas of $400 \times 400 \mathrm{~m}^{2}$ and $600 \times 600 \mathrm{~m}^{2}$ with 40 and 90 vehicles respectively. Simulation performance results for previously used $2 \times 2 \mathrm{~km}^{2}$ model with 200 vehicles $\left(50\right.$ vehicles $/ \mathrm{km}^{2}$ ) [18] suggest that provided the LTE network coverage is the same as $600 \times 600 \mathrm{~m}^{2}$ area, performance of 250 vehicles $/ \mathrm{km}^{2}$ can be evaluated in an area of $2 \times 2 \mathrm{~km}^{2}$ considering the performance degradation observed in the scaled areas. LTN velocity is set to $30 \mathrm{mph}$ while the HTNs are assumed to be moving at $20 \mathrm{mph}$, according to the city speed limits enforced in Glasgow city center.

We compare our scenario-I results with the previously proposed longest registration time algorithm implemented for traditional BUS-VANETs [14]. The primary performance measure used is the end-to-end message delivery delay. For scenarios-II and III, we evaluate the capacity of LTE network in terms of successful message delivery within the latency requirement set forth by standards and previous works.

\section{Simulation Results}

Vehicular networks have a fast changing topology due to their mobile nature. In the proposed MHAV framework, LTNs carry out V2V communications via HTNs. Therefore, the evaluation of experienced latency over such an architecture is vital. We first compare our message dissemination scheme employed by HTNs with the traditional BUS-VANET where the HTNs act merely as road side units (RSUs). Fig. 3 shows the cumulative density function (CDF) for the end-to-end delay for the scenario-I described in Section II-C1. Probability of message dissemination delay between LTNs via HTNs to be less than or equal to $50 \mathrm{~ms}$ is above $90 \%$ for the proposed scheme. 


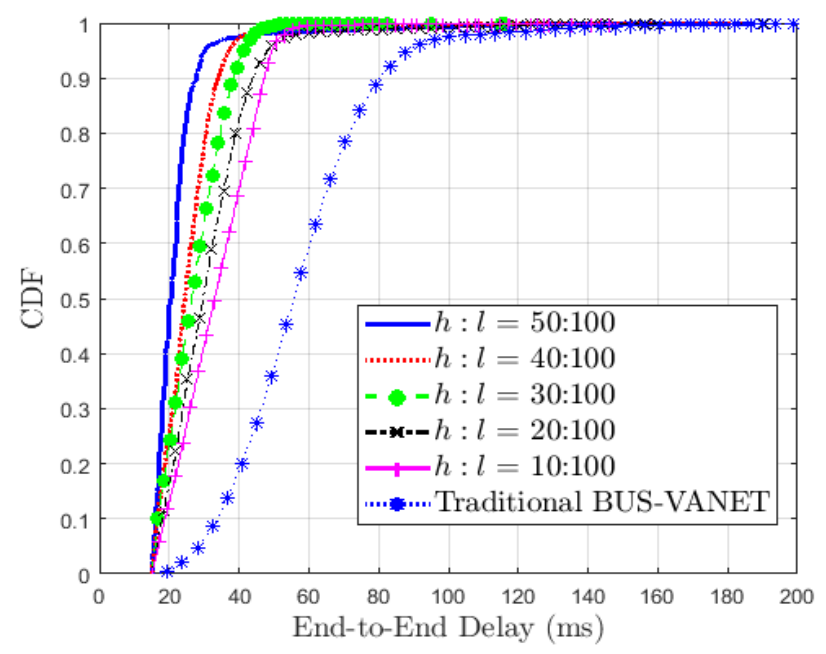

Fig. 3. Scenario-I: HTN - LTN Message Dissemination End-to-End Delay

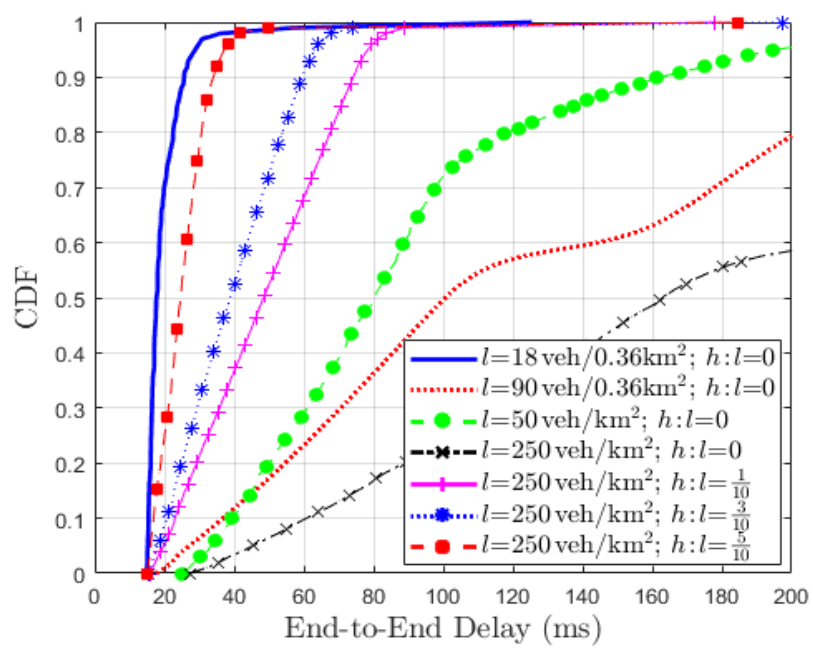

(a)

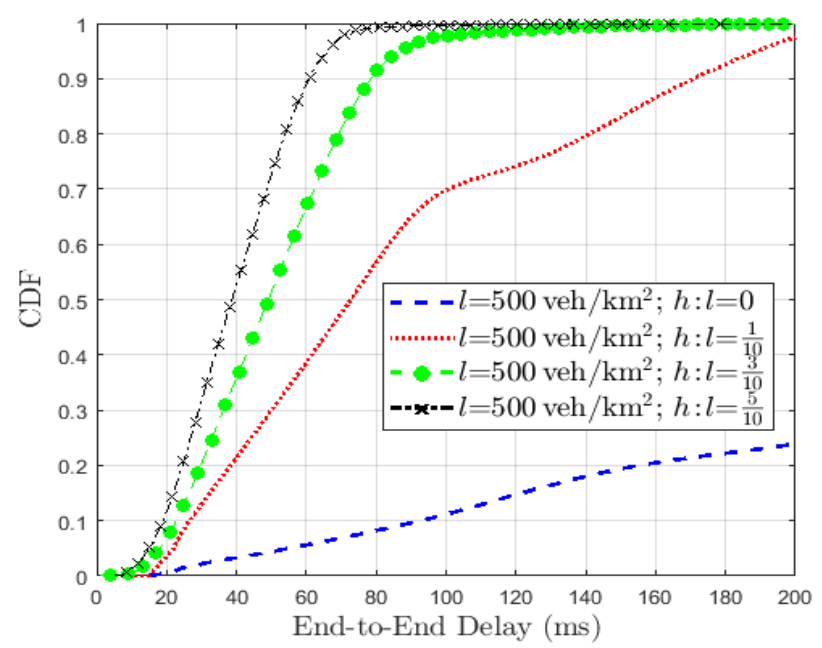

(b)

Fig. 4. Scenario-II \& III: LTE End-to-End Delay in $2 \times 2 \mathrm{~km}^{2}$ Glasgow City Center MHAV Framework (a) For $250 \mathrm{LTNs} / \mathrm{km}^{2}$, (b) For $500 \mathrm{LTNs} / \mathrm{km}^{2}$.

Comparing with the traditional BUS-VANET, a gain of about $50 \%$ is observed in the probability of end-to-end delay being $\leq 50 \mathrm{~ms}$. It can also be observed, that increasing the number of HTNs, performance improves. With 10 HTNs for 100 LTNs, this probability of end-to-end delay being $\leq 50 \mathrm{~ms}$ is $90 \%$. While increasing the number of HTNs to 50 for 100 LTNs, an increment of $8 \%$ can be observed. The reason for better performance is the resource utilization criteria, restricting the transmission area to the required awareness range. This approach leads to less capacity blocks and eventually lower broadcast flooding. Traditional BUS-VANETs propose performance improvements by adding RSUs in the network. While we propose to incorporate authority owned vehicles, which include public buses, council lorries, taxis, police patrolling cars, etc in MHAV architecture. This increases the number of HTNs, hence improving the network performance.

Furthermore, traditional BUS-VANETs face more delays due to the frequent switching between HTNs due to their proposed longest registration time algorithm. The amount of registration switching between HTNs is required to be low for the network to be robust and low latent. We tackle this problem in the registration criteria, which enables the HTN to reject registration in case of overloading and determine the highest connection delivery delay $(T)$ respectively.

Next we analyze scenarios II and III, where LTNs are either offloaded on to HTNs or if the HTN is not available, LTNs communicate via the LTE mobile network. Fig. 4 shows the CDF for end-to-end delay in $2 \times 2 \mathrm{~km}^{2}$ Glasgow city center employing the proposed MHAV framework. In order to establish a design criteria, a highly dense urban environment is investigated. Densities where the network gets heavily loaded are evaluated, while increasing the number of HTNs to satisfy the end-to-end delay requirement.

Fig. 4a shows the CDF of end-to-end delay experienced by 18 and 90 vehicles in $600 \times 600 \mathrm{~m}^{2}$ along with 200 vehicles in an area of $2 \times 2 \mathrm{~km}^{2}\left(50 \mathrm{veh} / \mathrm{km}^{2}\right)$. With the system degradation observed for increasing density of 18 to $90 \mathrm{veh} / 0.36 \mathrm{~km}^{2}$, results for 1000 LTNs $\left(250 \mathrm{veh} / \mathrm{km}^{2}\right)$ are obtained. For the case where there is no HTN present, the network with $250 \mathrm{veh} / \mathrm{km}^{2}$ is observed to be choked with probability of end-to-end delay being $\leq 50 \mathrm{~ms}$ at less than $10 \%$. However, when there is 1 HTN for every 10 LTNs present in the network, this probability goes up by $40 \%$. Furthermore, it can also be observed that by increasing the number of HTNs, the performance in term of latency improves. With $5 \mathrm{HTNs}$ for every $10 \mathrm{LTNs}$ in the network, the probability of end-to-end delay being $\leq 50 \mathrm{~ms}$ further improves to $98 \%$.

As expected, increasing the density of vehicles in the network leads to further performance degradation. Fig. 4b shows the end-to-end delay probability for a density of 500 vehicles $/ \mathrm{km}^{2}$. It is seen that with no HTN present, the end-to-end delay probability of less than $50 \mathrm{~ms}$ is only $6 \%$. Even with HTN to LTN ratio of $1: 10$, the network does not accommodate vehicular communications. However, with this ratio at 5:10, the probability for delay to be $\leq 50 \mathrm{~ms}$ is around $75 \%$. Therefore, it can be deduced that even with centralization and use of heterogeneous networks, high density networks pose capacity problems. Nevertheless, for a density of 250 vehicles $/ \mathrm{km}^{2}$, the proposed framework outperforms traditional vehicular networks.

\section{CONClusion And Future Work}

This paper proposes a systematic approach for designing a multitier heterogeneous adaptive VANET (MHAV) framework 
which consists of HTNs and LTNs. All the vehicles are assumed to have LTE and DSRC capabilities while LTNs register with HTNs to enable V2I and V2V communications over DSRC while the HTNs connect to the LTE network in order to provide infrastructure communications to its registered LTNs. A fall-back to LTE SAI in the case where there's no HTN present for registration is also proposed. Having authority owned HTN gateways tend to make the network more secure and addresses the privacy issue raised by many private car owners. Extensive system level simulations are carried out in Glasgow city center, a dense urban environment, in order to evaluate our proposed scheme. Results show that the proposed framework outperforms the traditional BUS VANET by almost $50 \%$ in terms of probability of end-to-end delay being $\leq$ $50 \mathrm{~ms}$.

We also evaluate a scaled up model of $2 \times 2 \mathrm{~km}^{2}$ with 1000 LTNs communicating via either HTNs or LTE mobile network. Gain of about $40 \%$ in terms of delay probability being $\leq 50 \mathrm{~ms}$ is observed when the HTN to LTN ratio is $1: 10$. This $50 \mathrm{~ms}$ delay probability is further improved to $98 \%$ with the HTN to LTN ratio of 5:10. Therefore, having heterogeneous networks incorporating our proposed multitier framework improves system performance in terms of latency, eventually paving way for L-5 vehicle automation.

In the future, we plan to investigate highway scenarios with MHAV, where due to the less frequent change in topology, certain parameters in our proposed scheme are speculated to be significant. LTE has been a promising candidate for vehicular networks. However, with the current growth in mobile users, catering for vehicular networks would require much more capacity. We also plan to investigate the impact of network slicing at $5 \mathrm{G}$ core for improving scalability and flexibility in vehicular networks.

\section{REFERENCES}

[1] SAE International, "J3016A: Taxonomy and Definitions for Terms Related to Driving Automation Systems for On-Road Motor Vehicles - SAE International," 2016. [Online]. Available: https://www.sae.org/standards/content/j3016_201609/

[2] "How Will Vehicle-To-Vehicle Communication Drive The Future Of Car Technology?" $2016 . \quad$ [Online]. Available: https://www.trafficsafetystore.com/blog/vehicle-to-vehiclecommunication/

[3] E. P. Dennis, A. Spulber, V. Sathe Brugeman, R. Kuntzsch, and R. Neuner, "Planning for Connected and Automated Vehicles," Center for Automotive Research, Tech. Rep., 2017. [Online]. Available: http://www.cargroup.org/publication/planning-for-connectedand-automated-vehicles/

[4] O. Altintas, J. Misener, S. Shladover, R. Puvvala, and V. Garg, "Look Ma, No Hands: Intelligent Transportation and the Wireless Networks that Drive It," in IEEE Wireless Communications and Networking Conference. IEEE ComSoc, 2017. [Online]. Available: http://host.comsoc.org/freetutorial/anritsu27/anritsu27.html

[5] G. Remy, S.-M. Senouci, F. Jan, and Y. Gourhant, "LTE4V2X: LTE for a Centralized VANET Organization," in IEEE Global Telecommunications Conference. Houston, TX, USA: IEEE, Dec. 2011, pp. 1-6.

[6] Cisco, "Cisco Visual Networking Index : Global Mobile Data Traffic Forecast Update , 2016 - 2021,” Tech. Rep., 2017.

[7] A. Bazzi, C. Campolo, B. M. Masini, A. Molinaro, A. Zanella, and A. O. Berthet, "Enhancing cooperative driving in ieee 802.11 vehicular networks through full-duplex radios," IEEE Transactions on Wireless Communications, 2018.

[8] S. Ucar, S. C. Ergen, and O. Ozkasap, "Multi-hop cluster based IEEE 802.11p and LTE hybrid architecture for VANET safety message dissemination," IEEE Transactions on Vehicular Technology, vol. 65, no. 4, pp. 2621-2636, April 2016.

[9] Y. Yang, P. Wang, C. Wang, and F. Liu, "An eMBMS based congestion control scheme in cellular-VANET heterogeneous networks," in 17th International IEEE Conference on Intelligent Transportation Systems (ITSC), Oct. 2014, pp. 1-5.
[10] A. Bazzi, B. M. Masini, and A. Zanella, "Performance Analysis of V2V Beaconing Using LTE in Direct Mode With Full Duplex Radios," IEEE Wireless Communications Letters, vol. 4, no. 6, pp. 685-688, Dec 2015.

[11] B. Schoettle and M. Sivak, "A survey of public opinion about autonomous and self-driving vehicles in the US, the UK, and Australia," 2014.

[12] S. Taha and X. Shen, "A physical-layer location privacy-preserving scheme for mobile public hotspots in nemo-based vanets," IEEE Transactions on Intelligent Transportation Systems, vol. 14, no. 4, pp. 1665 1680, Dec 2013.

[13] Public warning system (PWS) requirements (release 13), 3GPP TS 22.268 V13.0.0, Dec. 2015.

[14] X. Jiang and D. H. C. Du, "Bus-vanet: A bus vehicular network integrated with traffic infrastructure," IEEE Intelligent Transportation Systems Magazine, vol. 7, no. 2, pp. 47-57, Summer 2015.

[15] T. Kitani, T. Shinkawa, N. Shibata, K. Yasumoto, M. Ito, and T. Higashino, "Efficient vanet-based traffic information sharing using buses on regular routes," in VTC Spring 2008 - IEEE Vehicular Technology Conference, May 2008, pp. 3031-3036.

[16] G. Li, M. Ma, C. Liu, and Y. Shu, "Routing in taxi and public transport based heterogeneous vehicular networks," in 2016 IEEE Region 10 Conference (TENCON), Nov 2016, pp. 1863-1866.

[17] B. Liu, D. Jia, J. Wang, K. Lu, and L. Wu, "Cloud-assisted safety message dissemination in vanet cellular heterogeneous wireless network," IEEE Systems Journal, vol. 11, no. 1, pp. 128-139, March 2017.

[18] S. Ansari, T. Boutaleb, S. Sinanovic, C. Gamio, and I. Krikidis, "MHAV: Multitier Heterogeneous Adaptive Vehicular Network with LTE and DSRC," ICT Express, vol. 3, no. 4, pp. 199-203, $\operatorname{dec} 2017$. [Online]. Available: https://www.sciencedirect.com/science/article/pii/S240595951730276X

[19] S. Ansari, T. Boutaleb, C. Gamio, S. Sinanovic, I. Krikidis, and M. Sánchez, "Vehicular Safety Application Identifier algorithm for LTE VANET server," in 2016 8th International Congress on Ultra Modern Telecommunications and Control Systems and Workshops (ICUMT), Oct 2016, pp. 37-42.

[20] S. Ansari, T. Boutaleb, S. Sinanovic, C. Gamio, and I. Krikidis, "Vehicular multitier gateway selection algorithm for heterogeneous VANET architectures," in 2017 Advances in Wireless and Optical Communications (RTUWO). IEEE, nov 2017, pp. 180-185. [Online]. Available: http://ieeexplore.iee.org/document/8228530/

[21] A. M. S. Abdelgader and W. Lenan, "The Physical Layer of the IEEE 802 . 11p WAVE Communication Standard : The Specifications and Challenges," in World Congress on Engineering and Computer Science, vol. II, 2014, pp. 22-24.

[22] "The Royal Society for the Prevention of Accidents Road Safety Factsheet: Electronic Braking systems Electronic Braking Systems Factsheet Brake Assist How it works and its advantages," RSPA Road Safety Factsheet, no. 248, 2017. [Online]. Available: www.rospa.com

[23] Intelligent transport systems (ITS); basic set of applications; part1 functional requirements, ETSI TS 102 637-1 V1.1.1, Sep. 2010

[24] Z.H. Mir, and F. Filali, "LTE and IEEE 802.11p for vehicular networking: a performance evaluation," EURASIP Journal on Wireless Communications and Networking, no. 1, pp. 1-15, 2014.

[25] A. Vinel, "3GPP LTE versus IEEE 802.11p/WAVE: which technology is able to support cooperative vehicular safety applications?" Wireless Communications Letters, IEEE, vol. 1, no. 2, pp. 125-128, 2012.

[26] S. Ansari, M. Sanchez, T. Boutaleb, S. Sinanovic, C. Gamio, and I. Krikidis, "SAI: Safety Application Identifier Algorithm at MAC Layer for Vehicular Safety Message Dissemination Over LTE VANET Networks," Wireless Communications and Mobile Computing - Accepted in Press, 2018. [Online]. Available: https://www.hindawi.com/journals/wcmc/aip/6576287/

[27] "TS\#28.530: Management of network slicing in mobile networks; Concepts, use cases and requirements," 2017. [Online]. Available: www.3gpp.org/DynaReport/28530.htm

[28] E-UTRA Base Station (BS) radio transmission and reception (Release 12), 3GPP TS 36.104 V12.10.0, Jan. 2016

[29] T. Cerqueira and M. Albano, "Routesmobilitymodel: Easy realistic mobility simulation using external information services," in Proceedings of the 2015 Workshop on Ns-3, ser. WNS3 '15. New York, NY, USA: ACM, 2015, pp. 40-46. [Online]. Available: http://doi.acm.org/10.1145/2756509.2756515

[30] Model library release $n s-3.2$, Ns-3 network simulator, 2015. [Online]. Available: https://www.nsnam.org/docs/models/html/lte-design.html

[31] G. T. . V8.2.0, Evolved Universal Terrestrial Radio Access (E-UTRA), Base Station (BS) radio transmission and reception (Release 8). 3GPP, May 2008. 